Supporting Information

\title{
Sustainable Visible Light-Driven Heck and Suzuki Reactions Using NiCu Nanoparticles
}

\section{Adorned on Carbon Nano Onions}

S. Fatemeh Mohammadi Metkazini ${ }^{\mathrm{a}}$ Zahra Khorsandia ${ }^{\mathrm{a}}$ Akbar Heydaria*, Rajender S. Varma ${ }^{\mathrm{b} *}$ ${ }^{a}$ Department of Chemical, Faculty of Sciences, Tarbiat Modares University, PO Box 14155-4838,

14117-13116 Tehran, Iran,

Email:heydar_a@modares.ac.ir.

${ }^{b}$ Regional Centre of Advanced Technologies and Materials, Czech Advanced Technology and Research Institute, Palacký University in Olomouc, 'Slechtitelů 27, 783 71, Olomouc, Czech Republic,

e-mail: Varma.Rajender@epa.gov

Total number of pages (including cover): 17

Total number of tables: 1

Total number of figures: 26

Supporting references: 4 


\section{Content:}

Experimental................................................................. 3

General remarks........................................................... 3

Synthesis of catalyst...................................................... 3

General procedure for the Heck reaction...................................... 3

General procedure for the Suzuki reaction...................................... 4

Characterization of the catalyst....................................................... 5

Figure 1S. The FT-IR spectra of the pure CNO, fresh and reused catalyst............S5

Figure $2 \mathrm{~S}$. The XRD patterns of pure $\mathrm{CNO}$ and final catalyst.....................S6

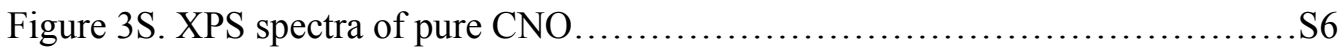

Table 1S. The data of BET nitrogen adsorption - desorption analysis...............S7

Figure $4 \mathrm{~S}$. The FE-SEM image of the reused catalyst............................. 7

Figure 5S. The TEM images of the reused catalyst................................ 7

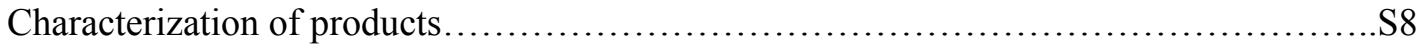

References.................................................................. 17 


\section{Experimental}

\section{General remarks}

All the chemicals used were purchased from Aldrich Chemical Company or Merck. The ${ }^{1} \mathrm{H}$ and ${ }^{13} \mathrm{C}$ NMR spectra were documented in $\mathrm{CDCl}_{3}$ on Bruker Avance, $400 \mathrm{MHz}$ and $100 \mathrm{MHz}$ respectively. The PerkinElmer FT-IR spectrophotometer was used for recording the FT-IR spectra. Transmission Electron Micrographs (TEM) were realized using Philips CM-200. FEI Talos F200X device equipped with an X-ray extreme field emission gun (X-FEG) - electron source module. was applied to record the high-resolution, analytical transmission electron microscopic (HRTEM) images. SEM images were recorded using FE-SEM JSM-7600F. A Bruker AXSDB Xray diffractometer using $\mathrm{Cu} \mathrm{K \alpha}$ radiations was used to record the X-ray diffractogram (XRD) in 2 theta range of $10-80^{\circ}$.

\section{Synthesis of catalyst}

To obtain carbon onions (CNOs), commercially available nano-diamond powders was utilized in the known procedure $[1,2]$. Briefly, it encompasses dispersion of nano-diamond and annealing at $1150{ }^{\circ} \mathrm{C}$ under a 1.1 MPa argon atmosphere. After one hour, the mixture was cooled to room temperature in final step and the ensued powder was heated at 400 . Next, to increase its surface area, the product was oxidized in air. The $\mathrm{NiCu}$ nanoparticles were prepared by the reduction of copper (II) nitrate trihydrate and nickel (II) nitrate

hexahydrate (Sigma Aldrich) according to the literature procedure [3, 4]. Purified CNOs (0.30 g) was dispersed into ethylene glycol $(100 \mathrm{ml}), 1.2 \mathrm{~g}$ of sodium hydroxide and $5 \mathrm{mmol}$ of metal precursors with a given molar ratio was added to the mixture; the reaction was performed at $180{ }^{\circ} \mathrm{C}$ for $4 \mathrm{~h}$. Subsequently, the mixture was cooled to room temperature and the final catalyst was separated by using an external magnetic bar and washed with hot ethanol. Finally, the catalyst was dried overnight at $80{ }^{\circ} \mathrm{C}$ in a vacuum oven.

\section{General procedure for the Heck reaction}


In a round-bottom flask equipped with a mechanical stirrer, a mixture of $\mathrm{K}_{2} \mathrm{CO}_{3}$ (2 eq.), olefin (1.1 mmol), and aryl halide $(1 \mathrm{mmol})$ in water $(3 \mathrm{~mL})$ was added to $20 \mathrm{mg}$ of the catalyst. The reaction was performed at room temperature conditions under the irradiation of a 300-W Xe lamp with wavelengths ranging from 400 to $800 \mathrm{~nm}$. The progress of the reaction was monitored by TLC (hexane/EtOAc, $80: 20$ ) and gas chromatography (GC). After the completion of the reaction, the mixture was diluted with dichloromethane and water. The organic layer was washed with brine, dried over anhydrous $\mathrm{MgSO}_{4}$, and concentrated under reduced pressure. The residue was purified by column chromatography. The products were characterized by comparing their physical properties, such as m.p., IR, ${ }^{1} \mathrm{H}$, and ${ }^{13} \mathrm{C}$ NMR spectra, with those reported in literature.

\section{General procedure for the Suzuki reaction}

In a round-bottom flask equipped with a mechanical stirrer, phenylboronic acid (1.2 mmol), aryl halide (1.0 $\mathrm{mmol}$ ), $\mathrm{K}_{2} \mathrm{CO}_{3}$ (2 eq.), and $20 \mathrm{mg}$ of the catalyst was added to the water $(3 \mathrm{~mL})$, the reaction was performed at room temperature under the irradiation of a 300-W Xe lamp with wavelengths ranging from 400 to 800 $\mathrm{nm}$. The progress of the reaction was monitored using TLC and GC. After the completion of the reaction, the mixture was diluted with dichloromethane and water. The organic layer was washed with brine, dried over anhydrous $\mathrm{MgSO}_{4}$, and concentrated under reduced pressure. The product was isolated by column chromatography to afford the corresponding products. The products were characterized by comparing their physical properties, such as m.p, IR, ${ }^{1} \mathrm{H}$, and ${ }^{13} \mathrm{C}$ NMR spectra with those reported in literature. 


\section{Characterization of the catalyst:}
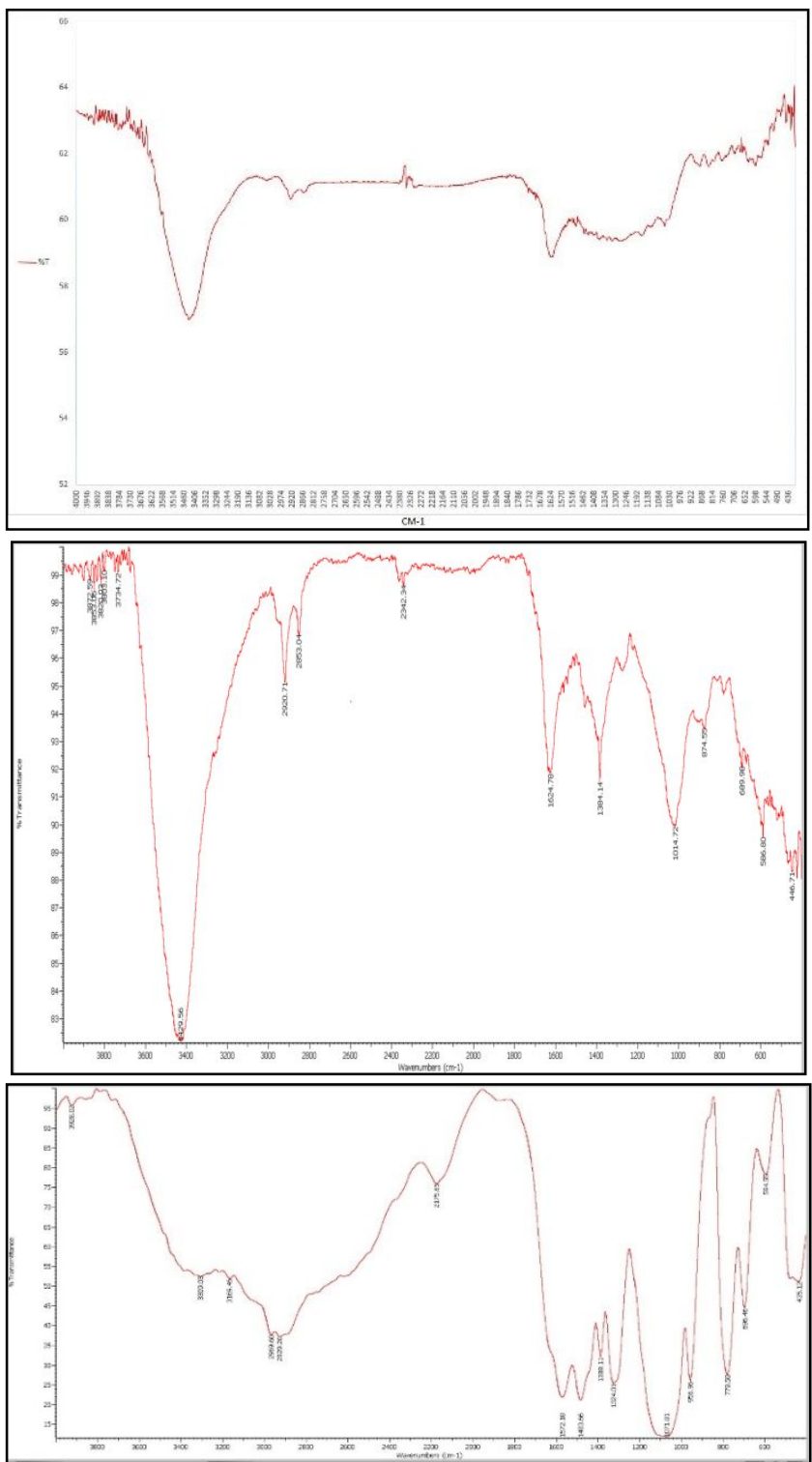

Figure S1. The FT-IR spectra of the pure CNO (above), fresh (middle) and reused catalyst (below) 

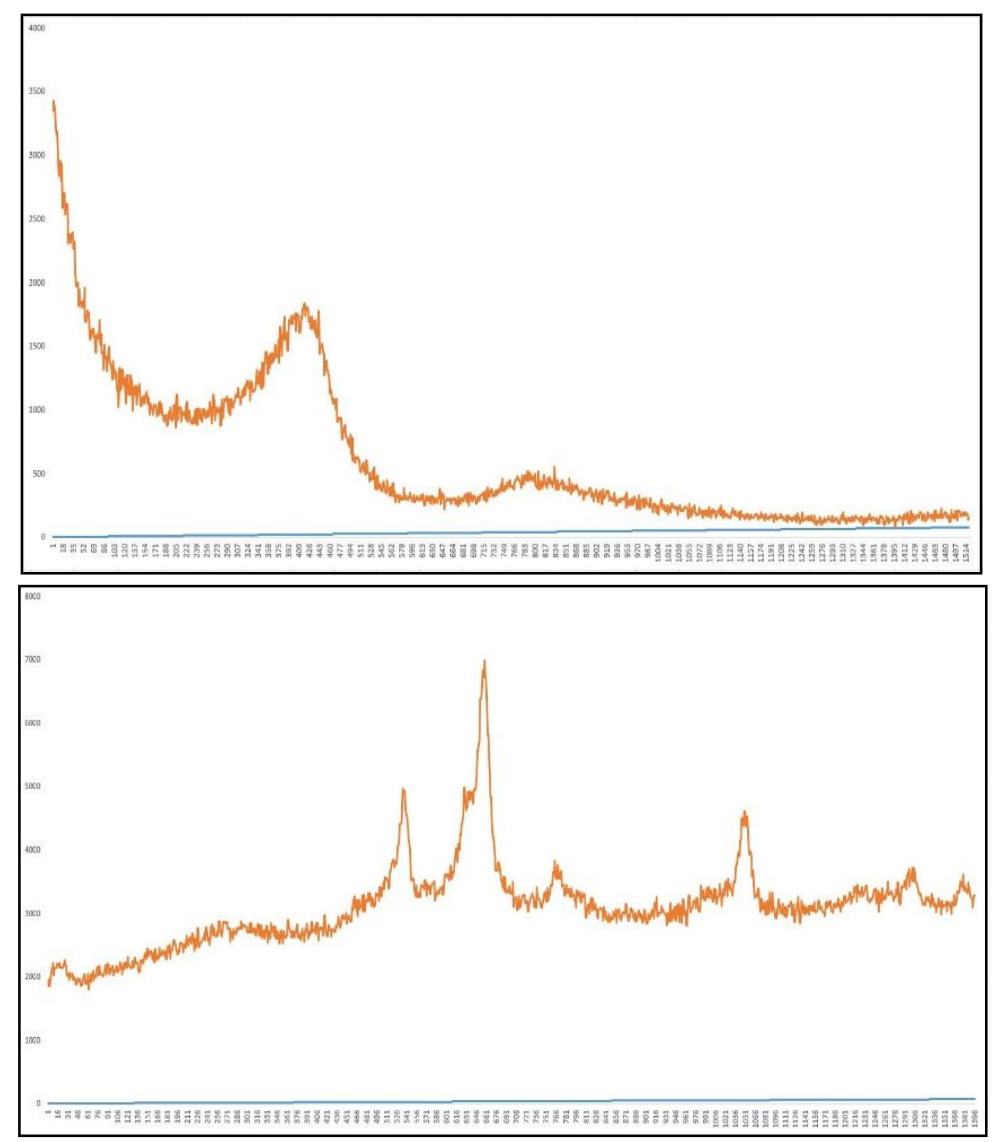

Figure S2. The XRD patterns of pure $\mathrm{CNO}$ (above) and final catalyst (below).

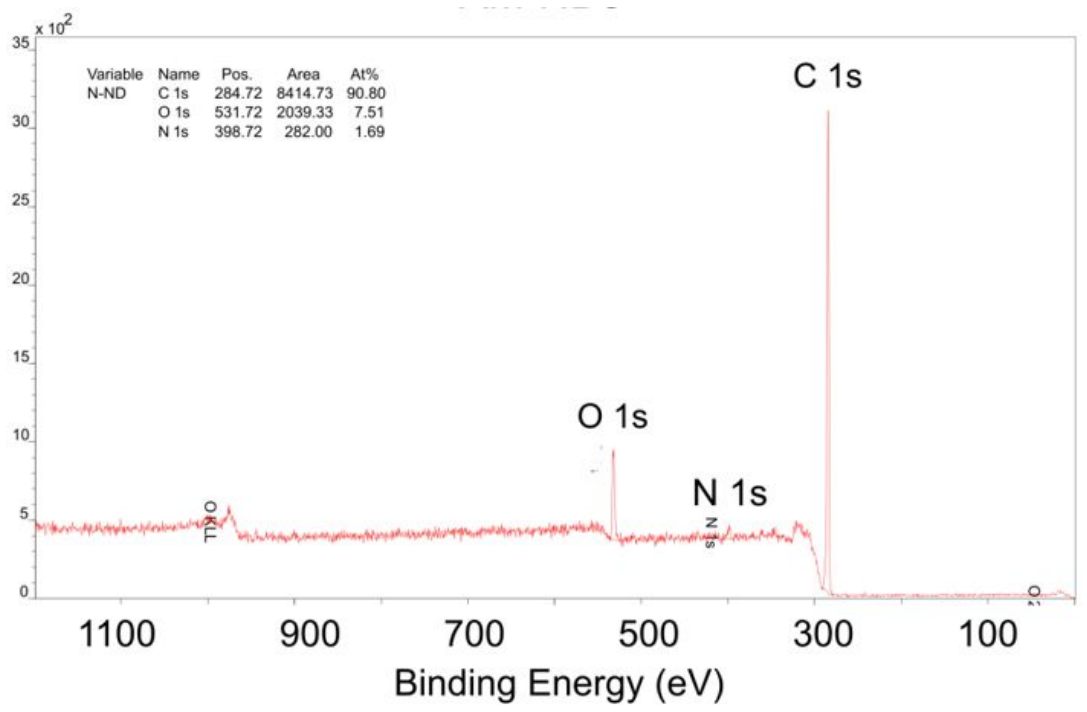

Figure S3. XPS spectra of pure CNO 


\begin{tabular}{|c|c|c|}
\hline \multicolumn{3}{|c|}{ Table S1. The data for BET nitrogen adsorption - desorption analysis } \\
\hline$V_{m}$ & 56.789 & {$\left[\mathrm{~cm}^{3}(\mathrm{STP}) \mathrm{g}^{-1}\right]$} \\
\hline $\mathrm{a}_{\mathrm{s}, \mathrm{BET}}$ & 247.17 & {$\left[\mathrm{~m}^{2} \mathrm{~g}^{-1}\right]$} \\
\hline$C$ & 77.608 & \\
\hline Total pore volume $\left(p / p_{0}=0.990\right)$ & 0.5101 & {$\left[\mathrm{~cm}^{3} \mathrm{~g}^{-1}\right]$} \\
\hline Mean pore diameter & 8.2553 & {$[\mathrm{~nm}]$} \\
\hline
\end{tabular}

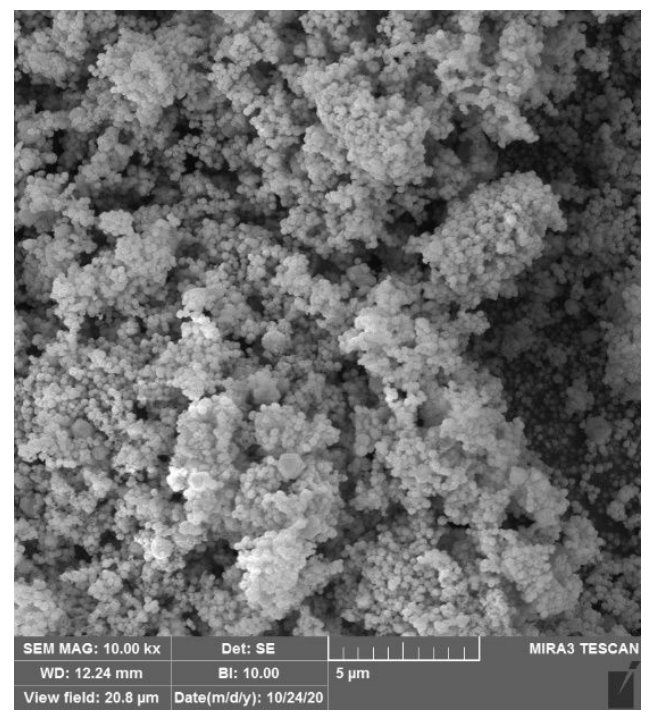

Figure S4. The FE-SEM image of the reused catalyst
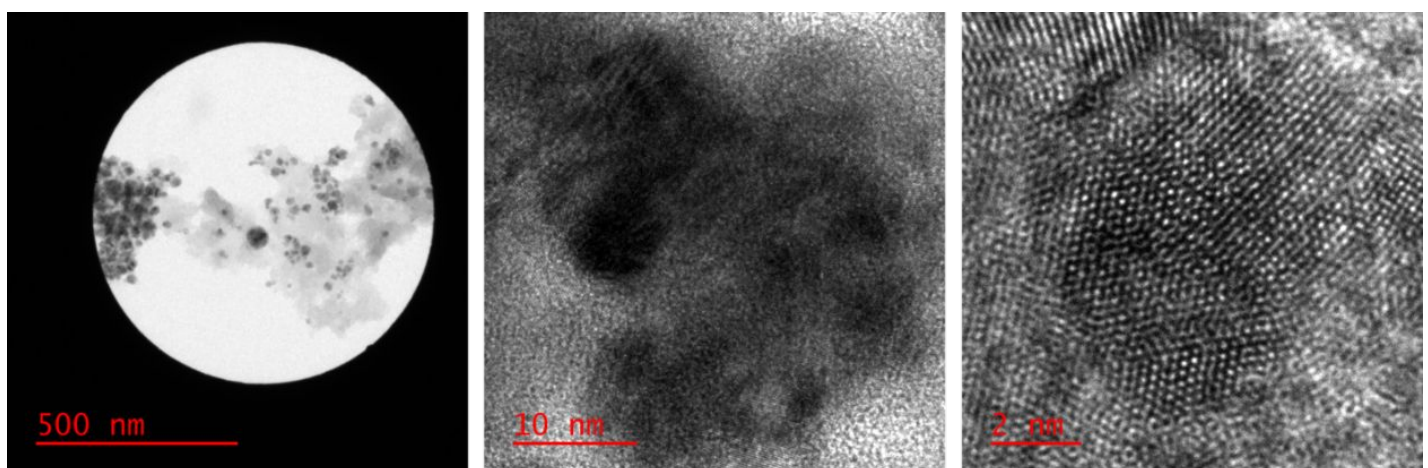

Figure S5. The TEM images of the reused catalyst. 


\section{Characterization of products:}

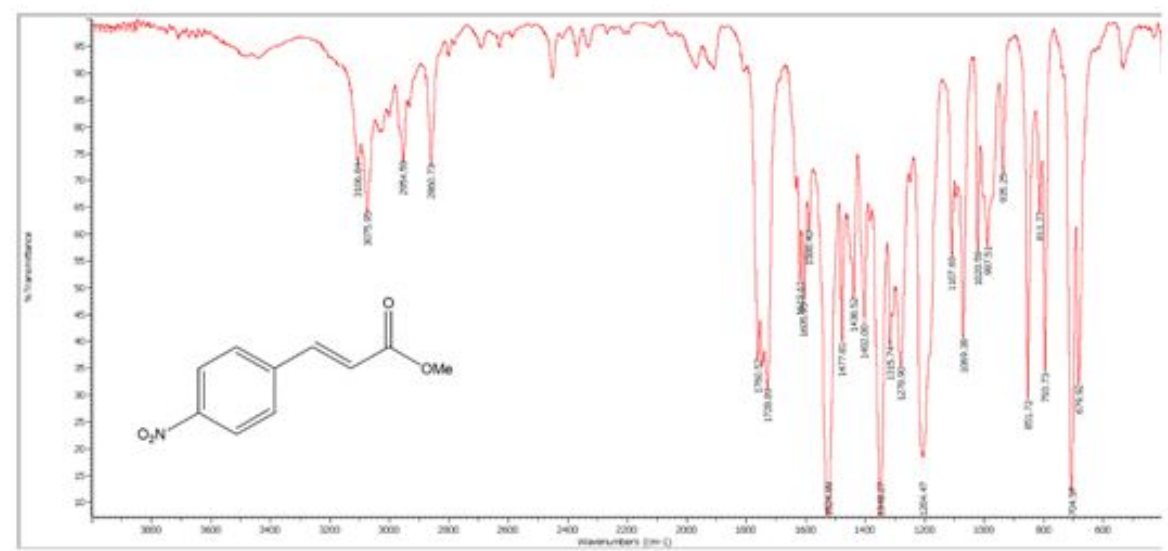

Figure S6. The FT-IR spectra of methyl 3-(4-nitrophenyl)acrylate.

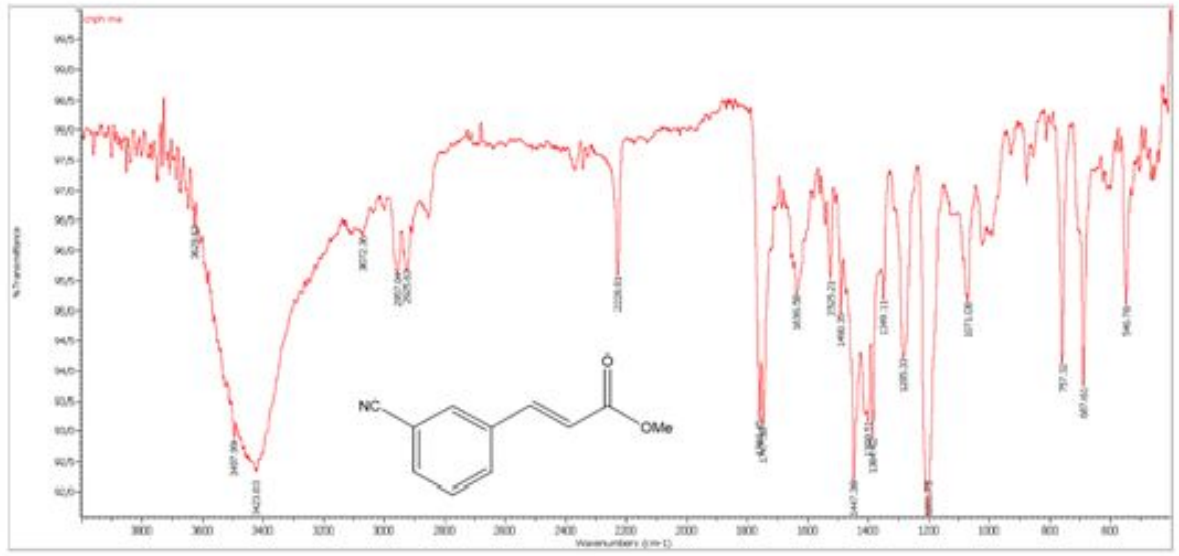

Figure S7. The FT-IR spectra of methyl 3-(3-cyanophenyl)acrylate. 


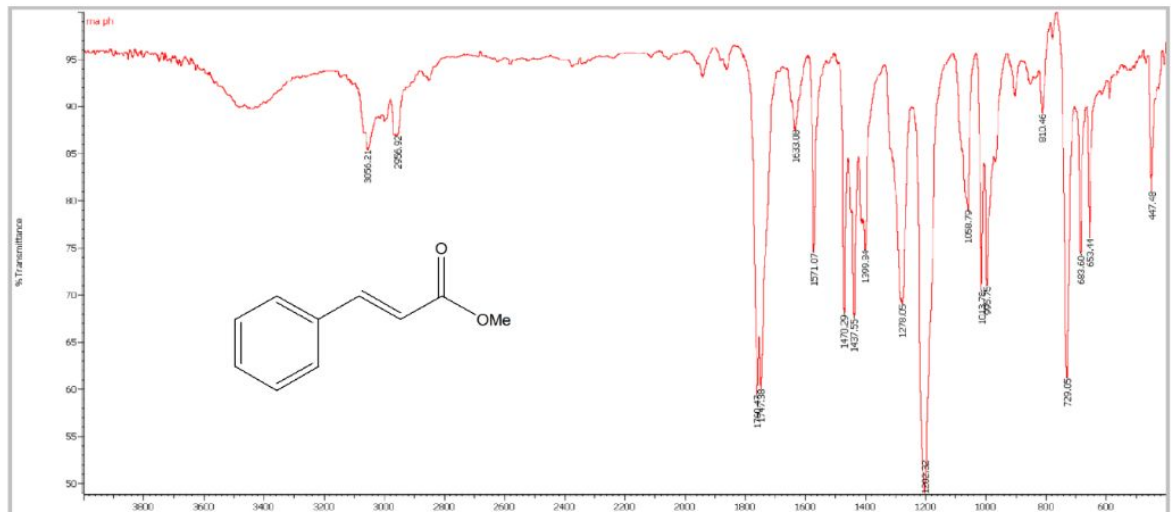

Figure S8. The FT-IR spectra of methyl cinnamate.

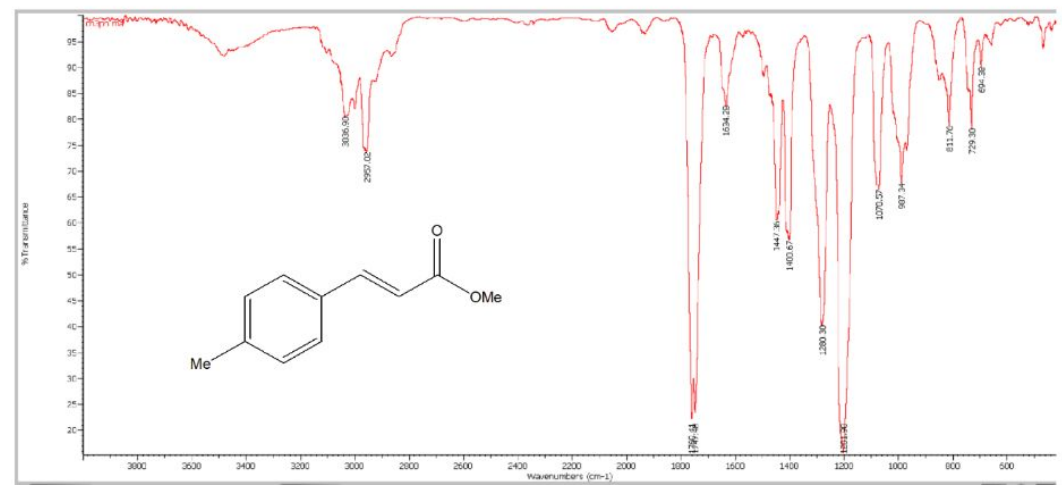

Figure S9. The FT-IR spectra of methyl 3-(p-tolyl)acrylate.

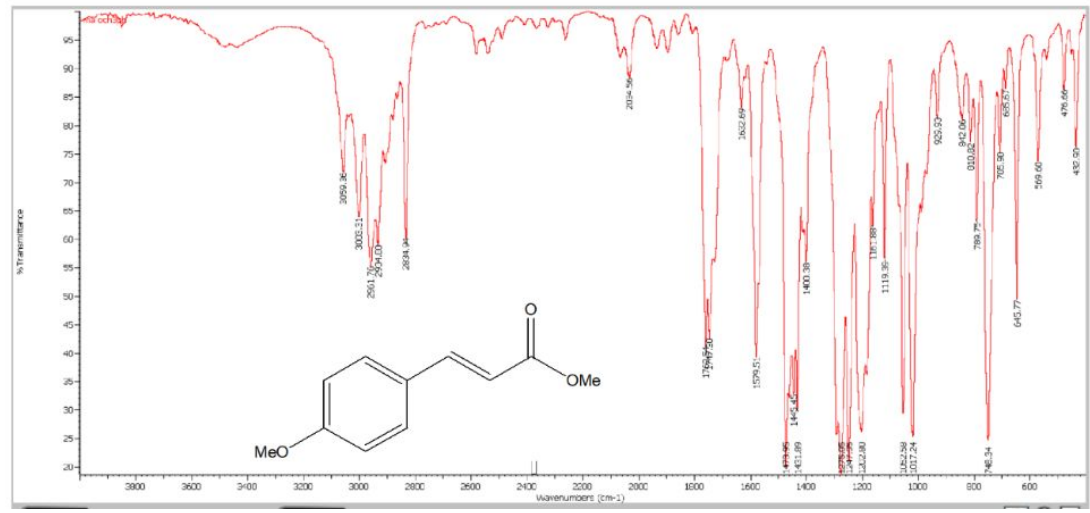

Figure S10. The FT-IR spectra of methyl 3-(4-methoxyphenyl)acrylate. 


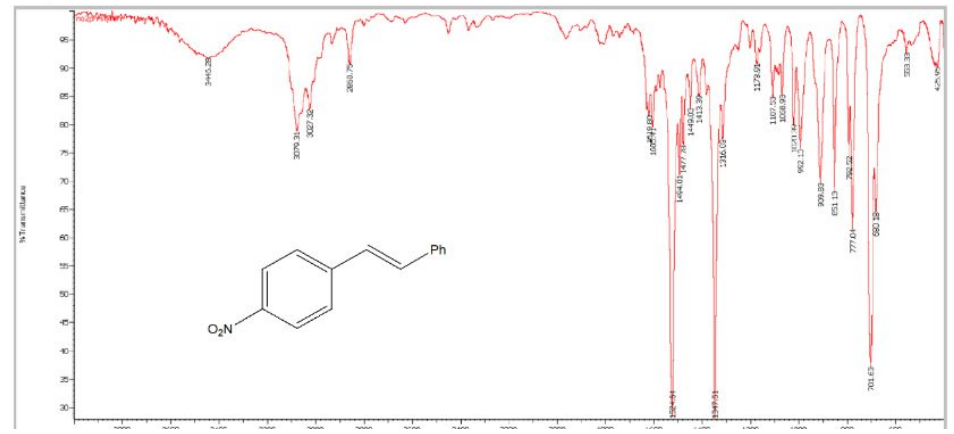

Figure S11. The FT-IR spectra of 1-nitro-4-styrylbenzene.

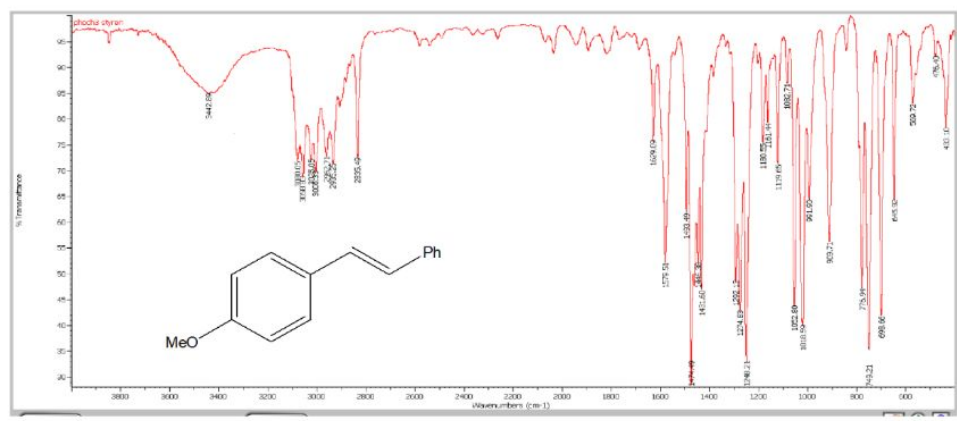

Figure S12. The FT-IR spectra of 1-methoxy-4-styrylbenzene.

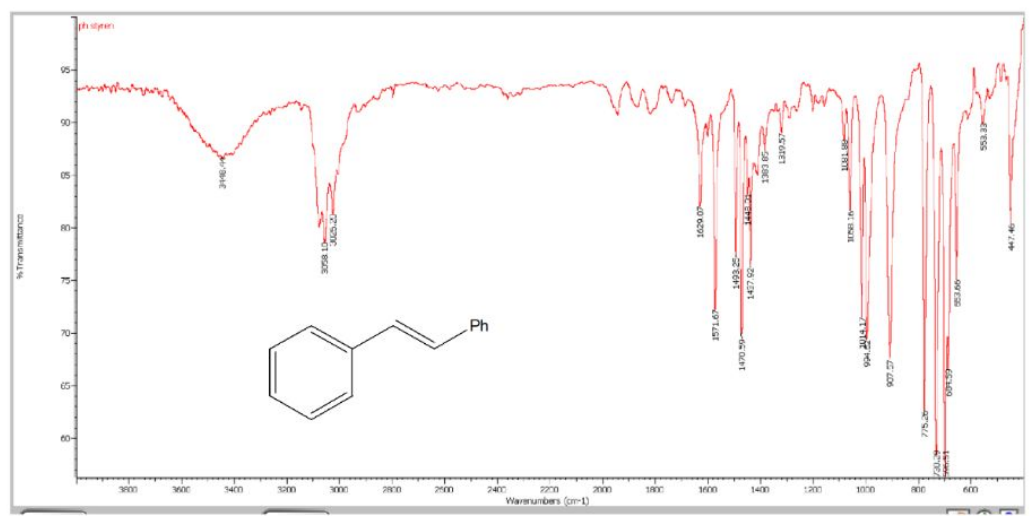

Figure S13. The FT-IR spectra of 1,2-diphenylethene. 


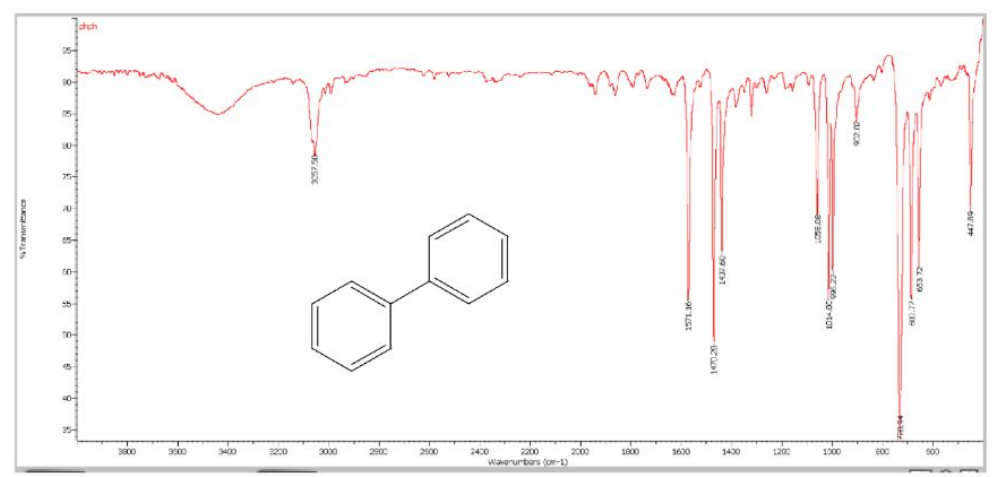

Figure S14. The FT-IR spectra of 1,1'-biphenyl

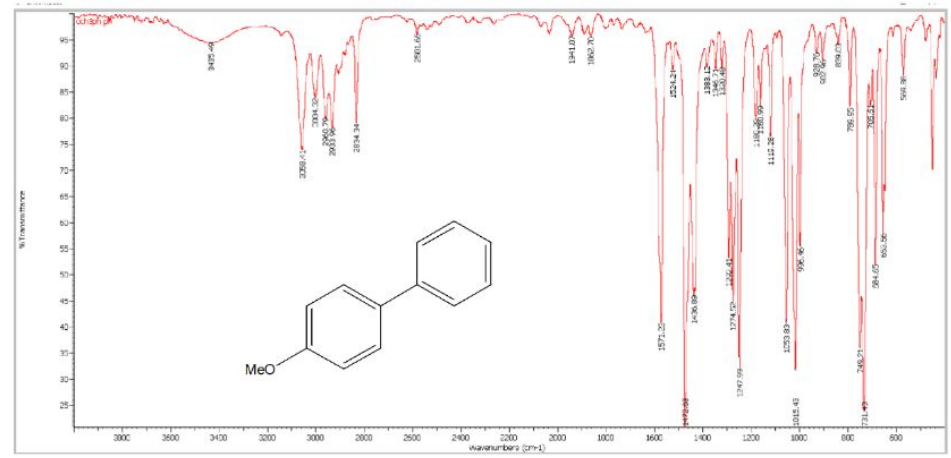

Figure S15. The FT-IR spectra of 4-methoxy-1,1'-biphenyl.

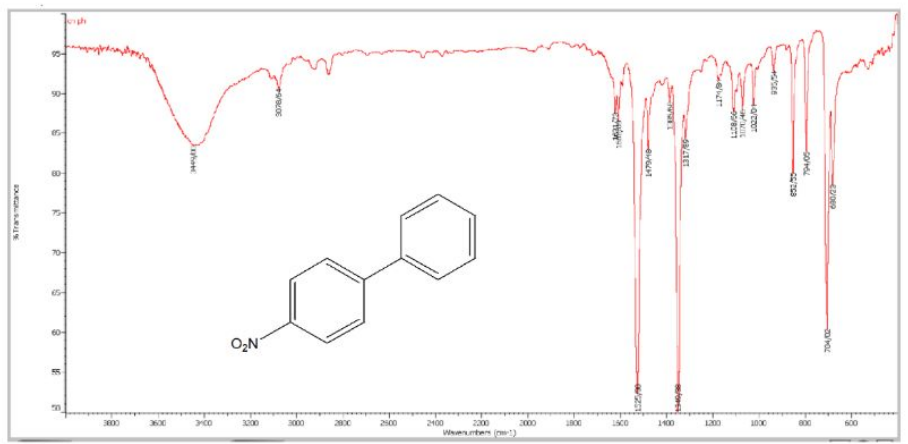

Figure S16. The FT-IR spectra of 4-nitro-1,1'-biphenyl. 


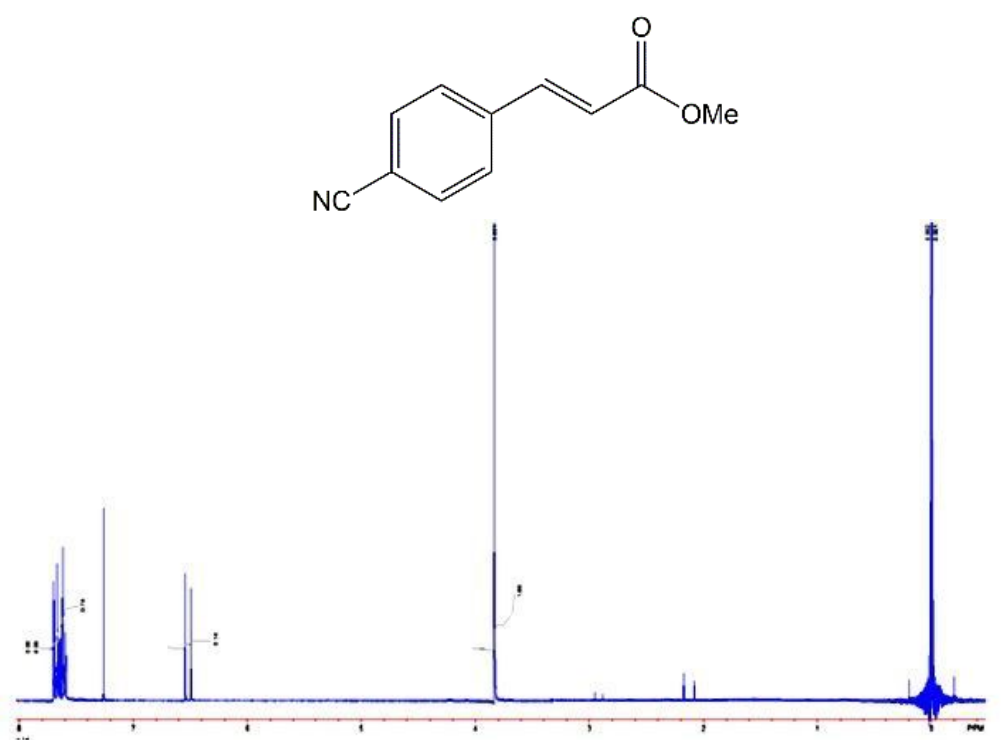

Figure S17. The ${ }^{1} \mathrm{H}$ NMR spectra of methyl 3-(4-cyanophenyl)acrylate.

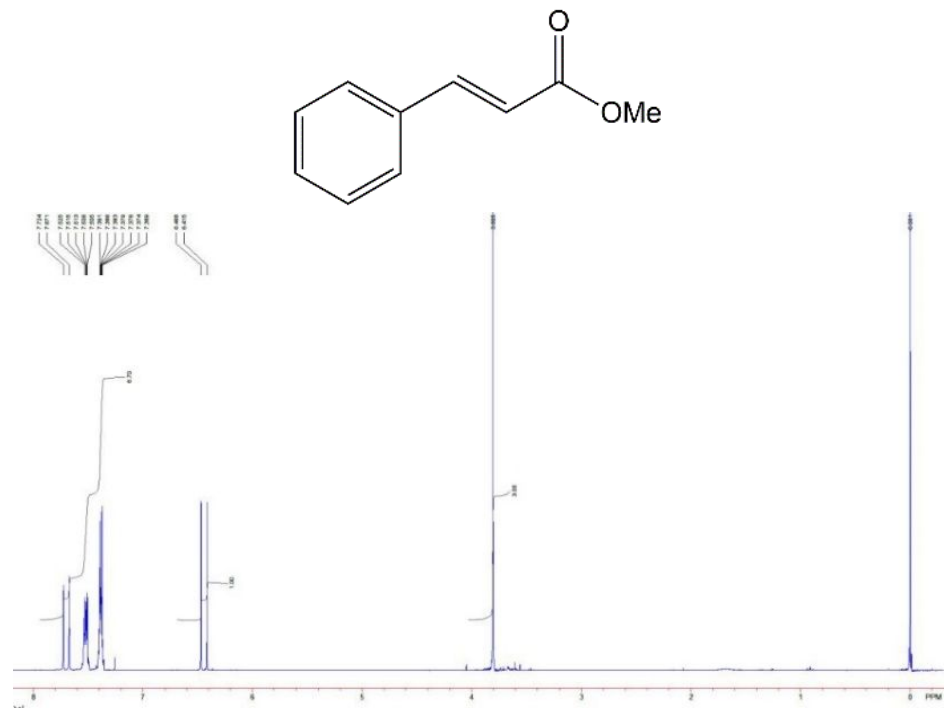

Figure S18. The ${ }^{1} \mathrm{H}$ NMR spectra of methyl cinnamate. 


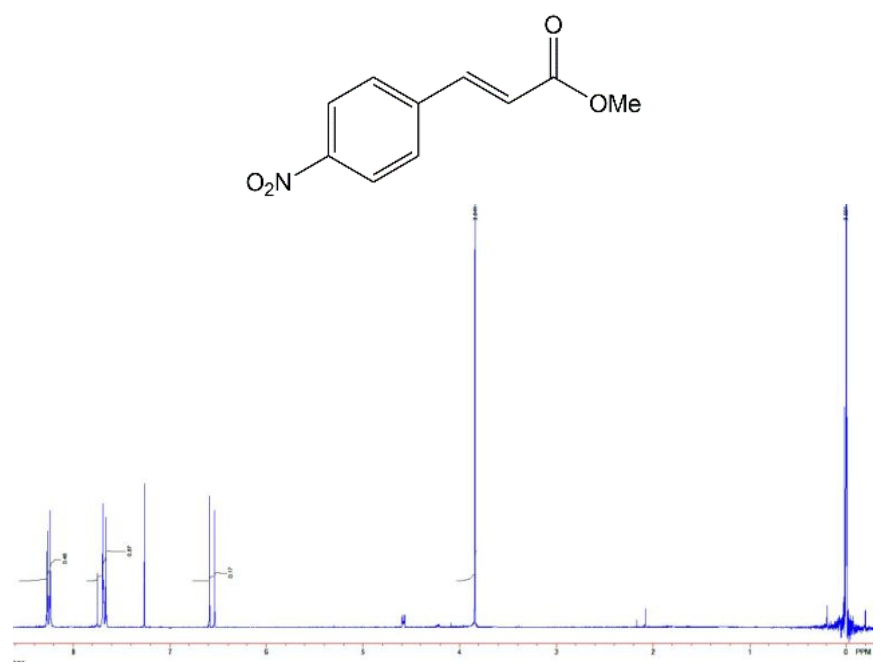

Figure S19. The ${ }^{1} \mathrm{H}$ NMR spectra of methyl cinnamate.

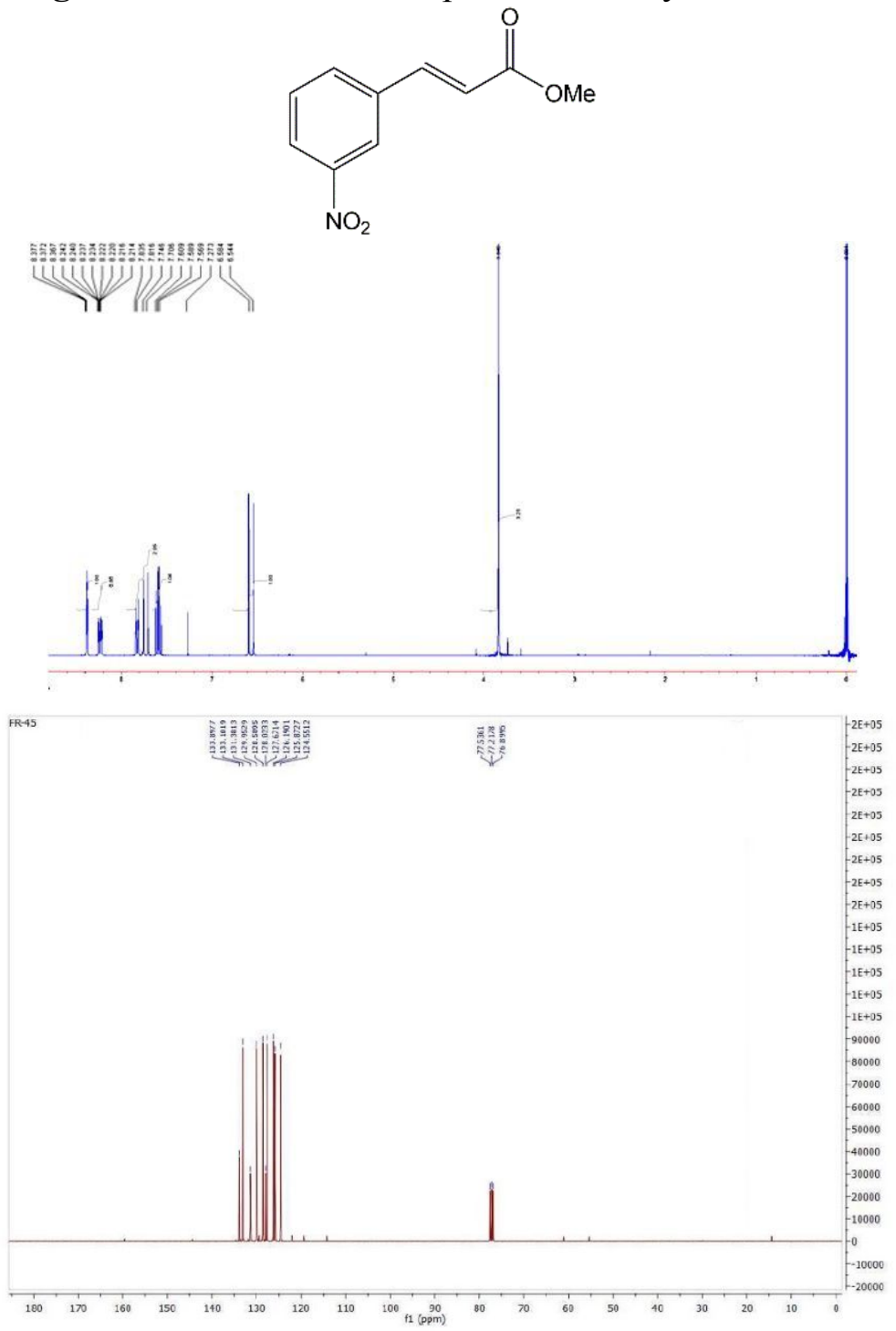

Figure S20. The ${ }^{1} \mathrm{H}$ and ${ }^{13} \mathrm{C}$ NMR spectra of methyl 3-(3-nitrophenyl)acrylate. 


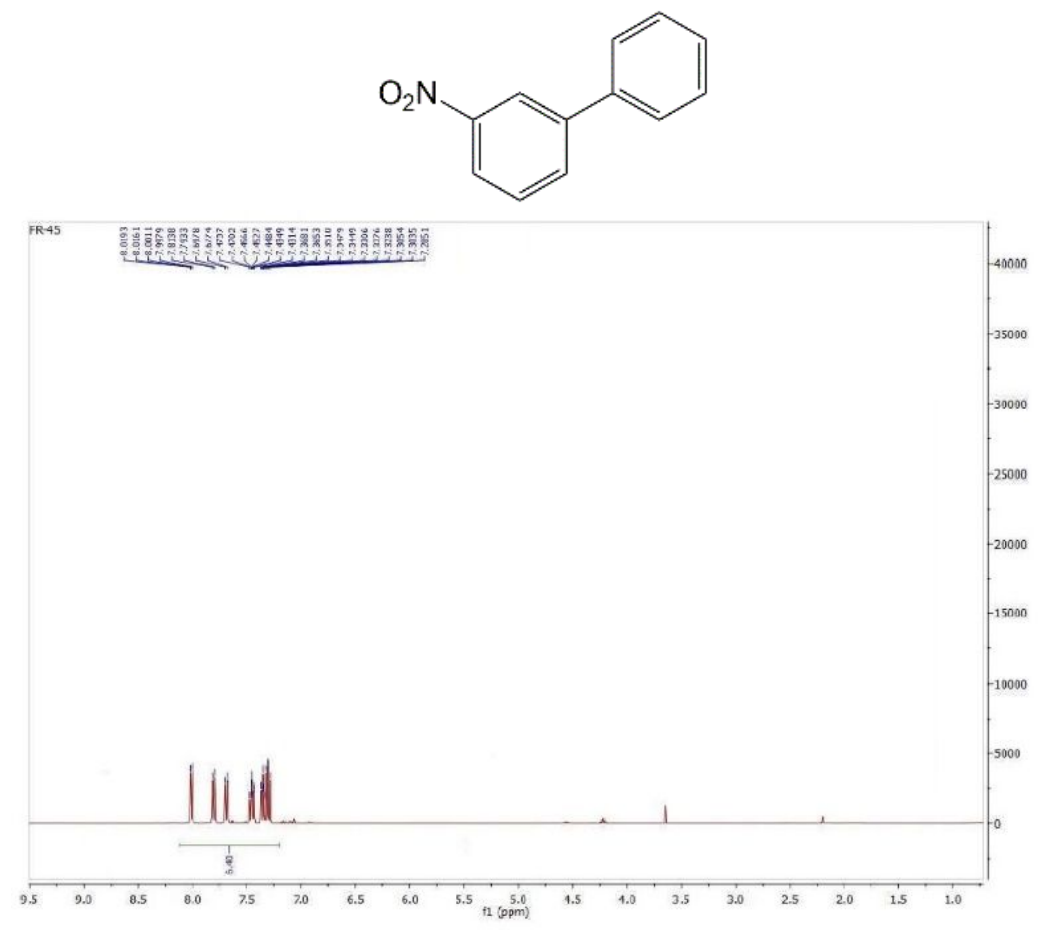

Figure S21. The ${ }^{1} \mathrm{H}$ NMR spectra of 3-nitro-1,1'-biphenyl.

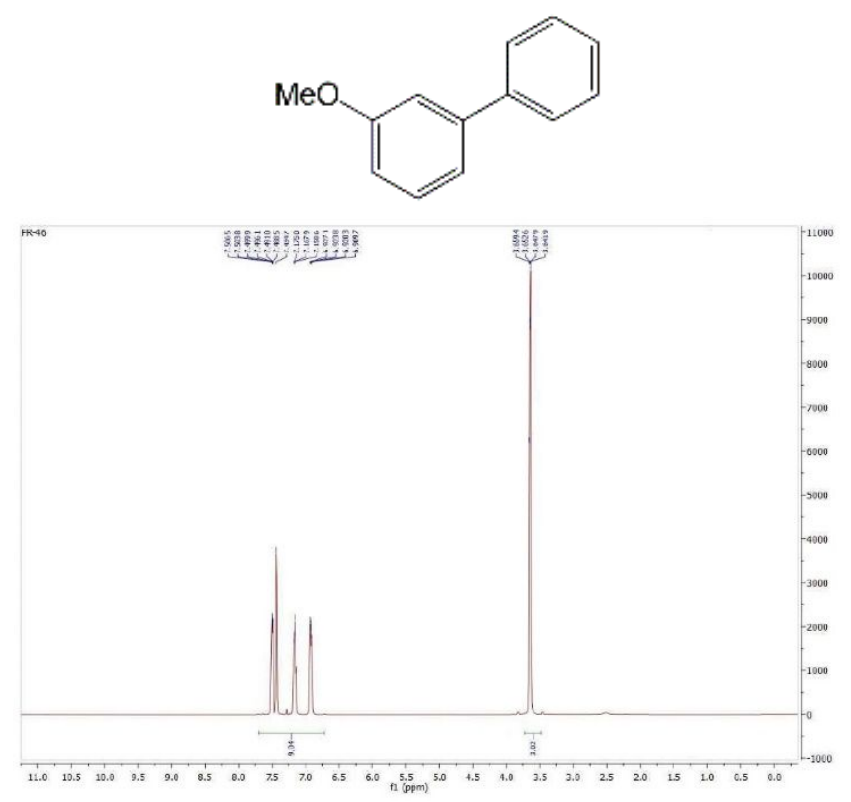

Figure S22. The ${ }^{1} \mathrm{H}$ NMR spectra of 3-methoxy-1,1'-biphenyl 


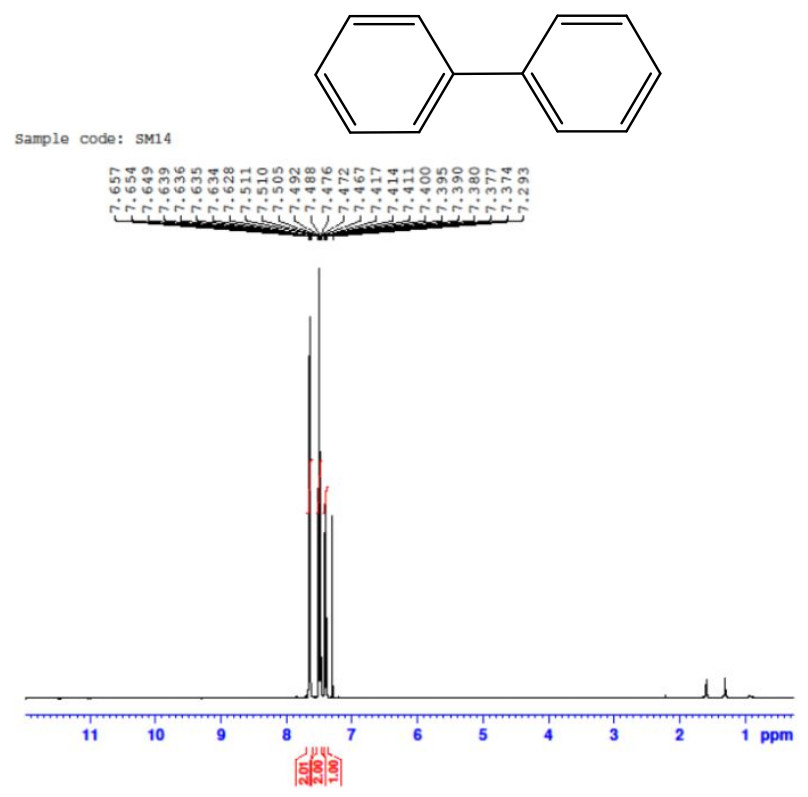

Figure S23. The ${ }^{1} \mathrm{H}$ NMR spectra of 1,1'-biphenyl.
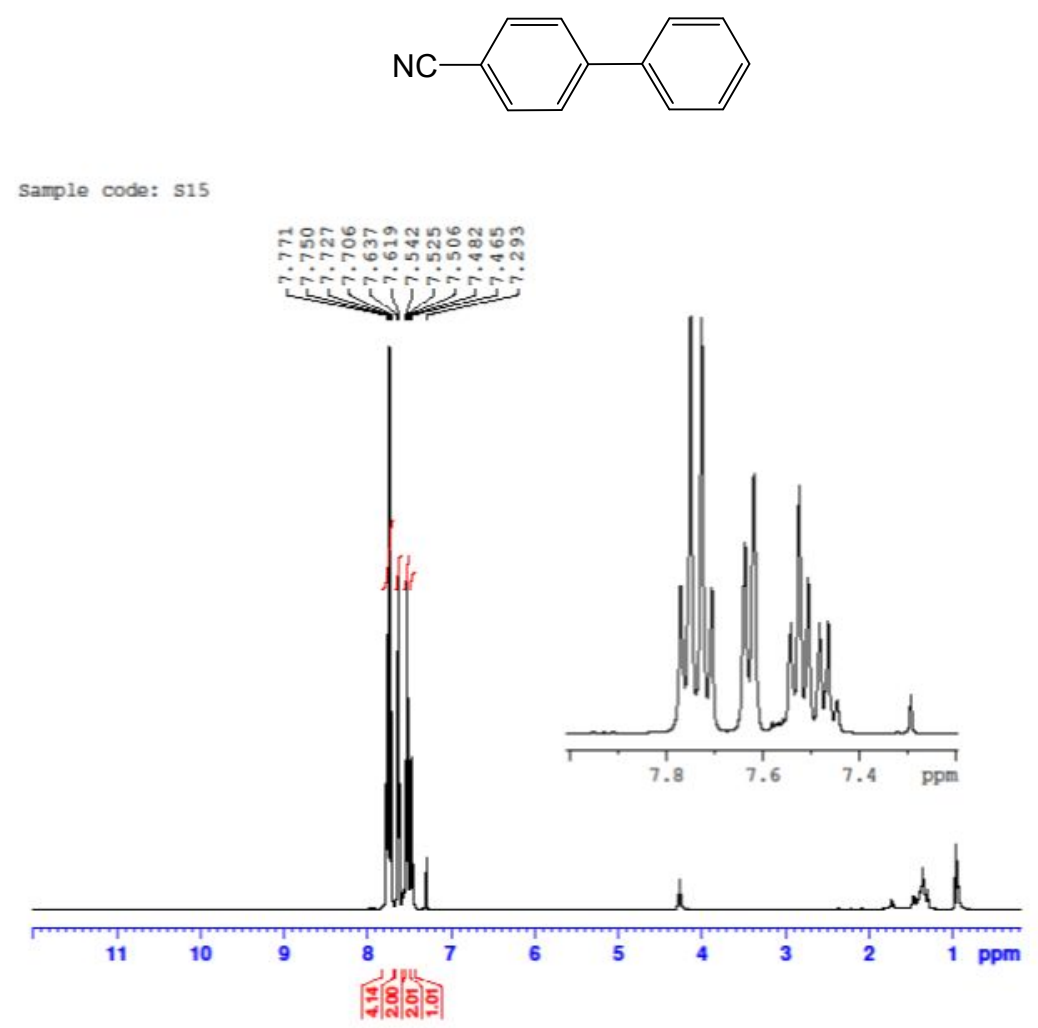

Figure S24. The ${ }^{1} \mathrm{H}$ NMR spectra of [1,1'-biphenyl]-4-carbonitrile. 


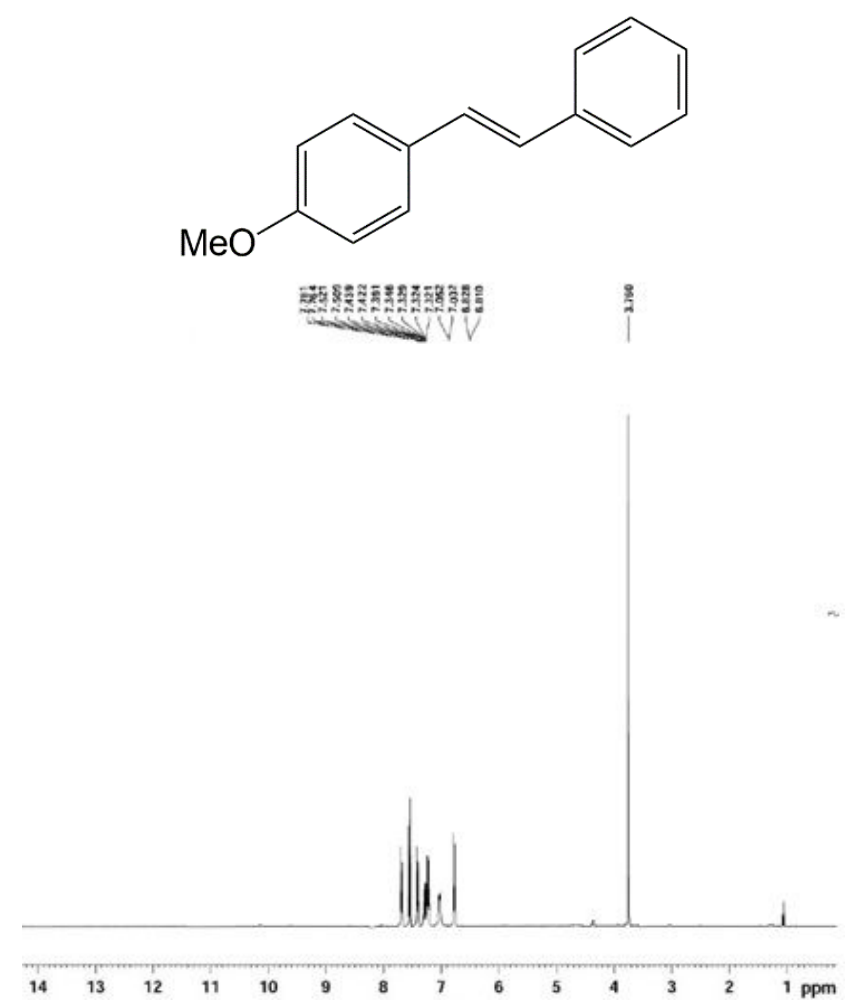

Figure S25. The ${ }^{1} \mathrm{H}$ NMR spectra of 1-methoxy-4-styrylbenzene.
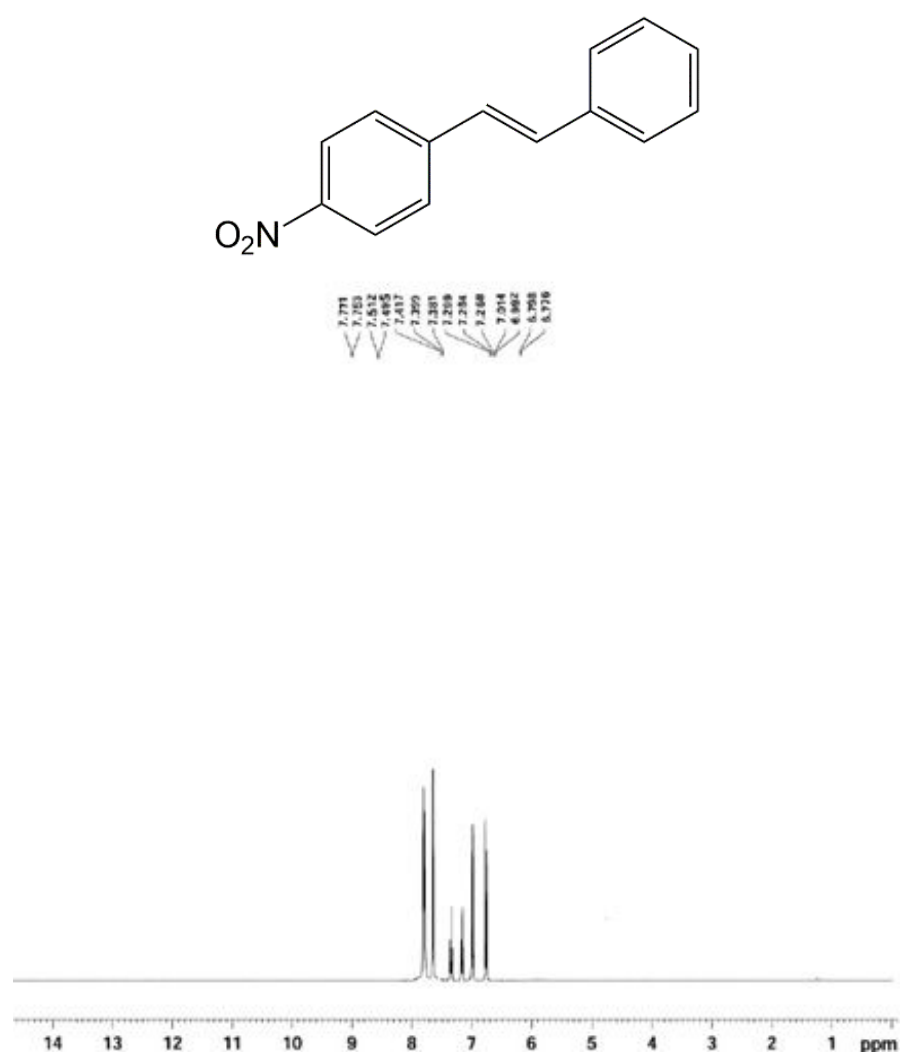

Figure S26. The ${ }^{1} \mathrm{H}$ NMR spectra of 1-nitro-4-styrylbenzene. 


\section{References:}

[1] V. Kuznetsov, Y.V. Butenko, Nanodiamond graphitization and properties of onion-like carbon, Synthesis, properties and applications of ultrananocrystalline diamond, Springer2005, pp. 199-216.

[2] S. Krishnamurthy, Y.V. Butenko, V. Dhanak, M. Hunt, L. Šiller, In situ formation of onion-like carbon from the evaporation of ultra-dispersed nanodiamonds, Carbon 52 (2013) 145-149.

[3] M. Zeiger, N. Jackel, M. Aslan, D. Weingarth, V. Presser, Understanding structure and porosity of nanodiamond-derived carbon onions, Carbon, 84, 2015, 584-598.

[4] Y. Shen, A. C. Lua, Synthesis of Ni and Ni-Cu supported on carbon nanotubes for hydrogen and carbon production by catalytic decomposition of methane, Applied Catalysis B: Environmental, 164, 2015, 61-69. 